
\title{
R Research Sourere \\ Characterization of Monoolein Langmuir Monolayers Spread at The Salt Solution/Air Interface
}

\section{Balaji Sopanrao Dhopte}

Sardar Vallabhbhai National Institute of Technology https://orcid.org/0000-0003-1576-1278

V. N. Lad ( $\nabla$ vnl@ched.svnit.ac.in )

SVNIT Surat: Sardar Vallabhbhai National Institute of Technology

\section{Research Article}

Keywords: Air-water interface, Compression isotherm, compressibility modulus, elastic modulus, Monoolein, Salt sub-phase

Posted Date: July 19th, 2021

DOI: https://doi.org/10.21203/rs.3.rs-158120/v3

License: (c) (1) This work is licensed under a Creative Commons Attribution 4.0 International License.

Read Full License 


\title{
Characterization of Monoolein Langmuir Monolayers Spread at the Salt Solution/air Interface
}

\author{
Balaji S. Dhopte, V.N. Lad* \\ Chemical Engineering Department, Sardar Vallabhbhai National Institute of Technology \\ (SVNIT), Surat Gujarat -395007, INDIA
}

\begin{abstract}
The Langmuir monolayer is commonly described at interfaces for an insoluble homogenous single molecular layer. Langmuir monolayers have demonstrated various soft matters and complex fluids by forming ideal uniform two-dimensional structures over the air-water interface. The Langmuir monolayer has advantages for evaluating physicochemical properties at interfaces and, for the insoluble molecules, can be applied simultaneously to the different interaction occurrences at interfaces. In this regard, monoolein lipid was used as a spreading solvent to create a Langmuir monolayer. Five different types of salt subphases were applied for the physicochemical properties interaction studies. On the airwater interface, the surface properties of monoolein lipids were investigated for interfacial phase behaviors using the Wilhelmy plate pressure sensor technique compression isotherm $(\pi-a)$. Data and analysis were also contributed to correspondent and precise verification of physical state behaviour with the surface pressure measurements on the interfaces through the compressibility modulus parameters on the surface. In the experiments, the interfacial activity of the monoolein lipids was found to be stable on the aqueous subphase. At the same time, the area per molecule over the interface did not affect much as a subphase with the change in various salts. The experiment's repeatability and reproducibility were affirmed by the difference in Langmuir monolayer's particular phase transition orientation behavior and the stability of colloidal lipid dispersion. However, Langmuir monolayer formation contributes to several special groups being restructured and found to be a more remarkable natural process for their attractive organic, dynamic structural properties over the interface. Still, the interfacial molecular dynamics have proved challenging to calculate.
\end{abstract}

Keywords: Air-water interface; Langmuir monolayer; Monoolein lipid; Phase transition; Salt subphases
*Corresponding author. V. N. Lad
Email:vnl@ched.svnit.ac.in
lad.vnl@gmail.com 


\section{Graphical Abstract:}

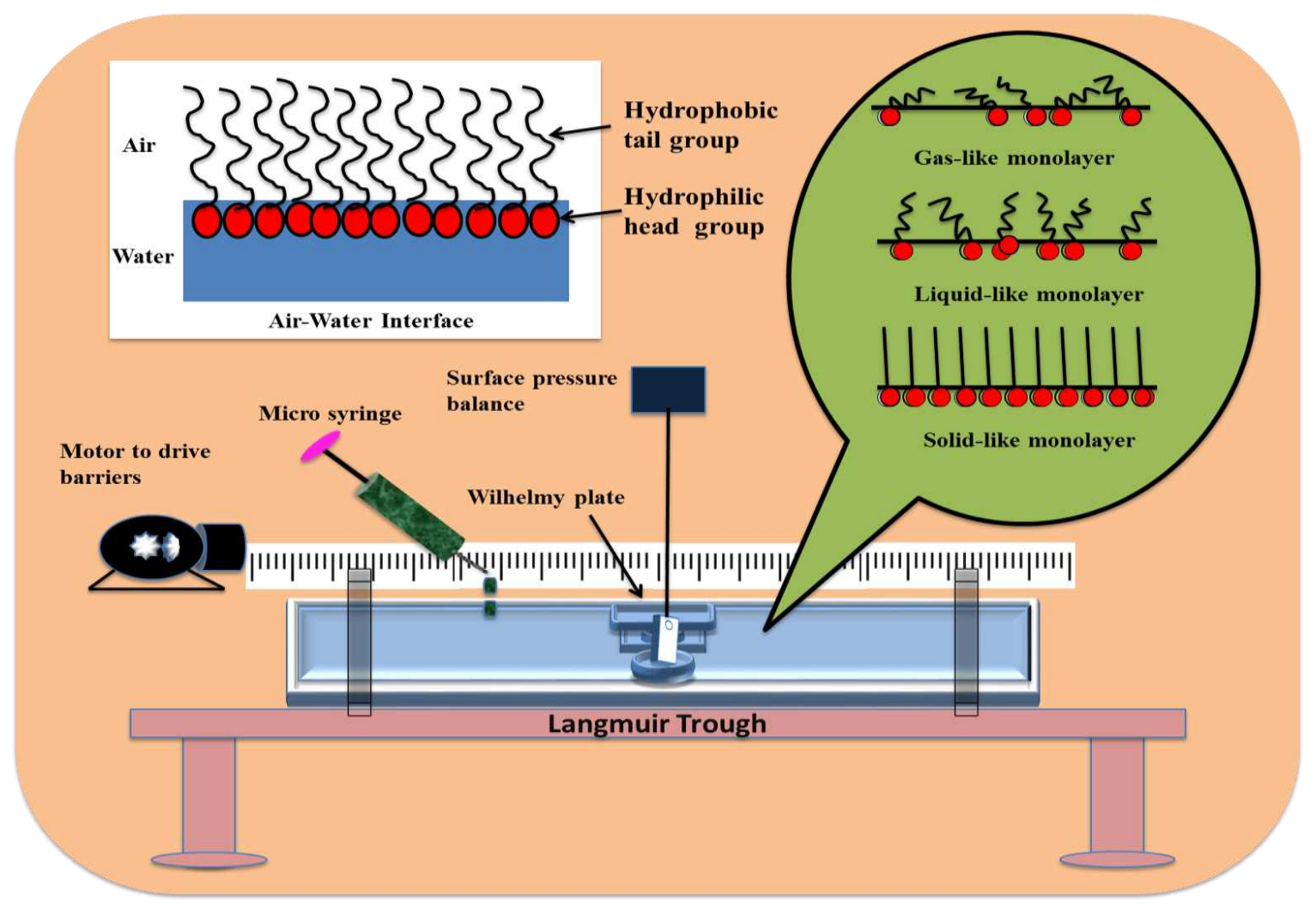

\section{Highlights:}

- Langmuir monolayer approach of surface pressure-area by molecules $(\pi$-a) interaction phase behaviour curves at the interface between air and water

- Wilhelmy plate surface pressure sensor is applied to monoolein lipid monolayer with different salt subphases changing

- Confirmation on phase behavioural changes with a determination of lipid compressibility modulus Langmuir monolayer

- The desired orientation and stability of colloidal dispersions distinguishes on various salt subphase interaction 


\section{Introduction}

The rising popularity of the Langmuir monolayer technique from 1920 to 2020 has enormously increased. In the past, Irving Langmuir's discovery was awarded the Nobel Prize for remarkable achievement in chemistry in 1932 [1]. Afterward, many researchers have studied various applications based on science and technology interfaces [2]. These applications include protein-lipid macromolecules [3], molecular thin films[4], colloids [5], nanomaterials [6], and more recently, monolayers as vehicles for bioactive macromolecules membrane [7]. Nowadays, the advancements carried out in the Langmuir monolayer technique were successfully utilized in Alzheimer's disease and the surface chemistry interaction of peculiar conditions $[6,8]$. During the Langmuir monolayer formation, the uniform distribution occurs, due to which the colloidal film description became more comfortable for the particle size identification and structural properties characterization [2,9]. The Langmuir monolayer's interfacial properties have become an attractive source for its ability to control accurate measurements of the homogeneous monolayer at the interfaces $[10,11]$. The difficulty lies in the surface pressure measurement properties for performing the real-time characterization of Langmuir monolayer experimentation [4,8]. Monoolein forms monolayers in the liquid expanded phase only, and no solid phase could be observed by the values of compression modulus [12,13].

Natural lipids can carry a negative net charge or are neutral, depending on the head group structure interacting with the aqueous phase [14]. Lipids are widely used for the biomembrane process, and they are primarily heterogeneous in chain length, chain saturation, and head group structure [15]. It is commonly used in the food industry and widely used for low fat and instant food mix surfactants in traditional foods [16-18]. Langmuir lipid monolayer formation is a bit straightforward since the second most essential macromolecule is after the protein bio-macromolecules. In molecular species, the lipid macromolecules are highly surface-active amphiphilic materials and are purely hydrophobic. Many researchers studied the monolayer approach of Langmuir lipid using compression isotherm [4,19]. The lipid monolayer macromolecule assists in the understanding of the folding unfolding behaviour of interacting molecules along with the structural analysis of the formed monolayers [20]. The elastic energy measurement was recently carried out with the lipid macromolecules' topographical changes by the Langmuir monolayer approach [21-24]. Similarly, several other theoretical methods were developed and used for the various experiments to understand better 
the driving force for folding unfolding phase transition behaviour observations. In addition, the heterogeneity of the molecules plays a significant role in determining the film's 3D structure and stability $[25,26]$.

A commonly known chemical name for monoolein lipid is 9-Octadecenoic acid (Z)monoester with 1,2,3-propanetriol and 1-Monoolein (1-(cis-9-Octadecenoyl)-rac-glycerol) is also familiar [27]. It is a viscous, pale yellow liquid with a characteristic odour in terms of physicochemical properties. From the point of view of the molecular structure shown below, it is composed of a hydrocarbon chain, which is attached by an ester bond to a glycerol backbone (see Figure 1). Macroscopically speaking, the moiety of glycerol gives polar characteristics to this part of the molecule for a hydrophobic head, which implies that the formation of hydrogen bonds with water in aqueous solutions by the hydrocarbon chain known as the hydrophilic tail, with a double bond position, is highly hydrophobic [39]. In water and cold alcohol, monoolein is insoluble but is regularly soluble in oil, petroleum ether, chloroform, and even hot alcohol. Since it is a non-toxic, biodegradable, and biocompatible material, monoolein lipids could have very desirable properties as in a food emulsion [28].

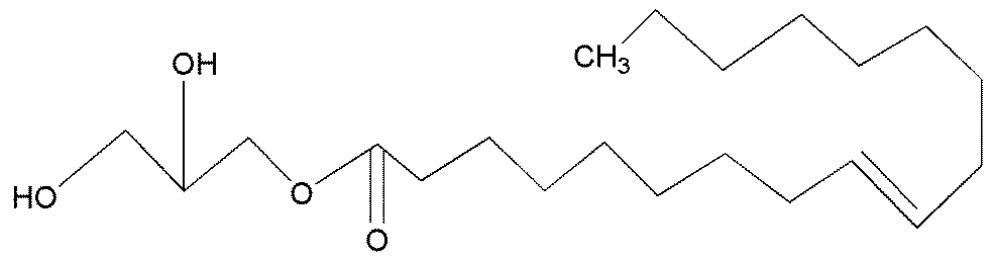

Figure 1. Chemical structure of monoolein lipid [27]

The significant interest in evaluating the lipid Langmuir monolayer technique on the air-water interface has increased to understand the lipid macromolecules interaction effect with the change in subphase containing liquids. The surface properties of monoolein lipid macromolecules were investigated in this paper by using the Wilhelmy plate surface pressure sensor at the air-water interface. For the phase behavioural orientation of the lipid molecules and their interaction with various ionic salt subphase molecules on an interface, compression isotherm $(\pi-a)$ was observed. The shift in the phase transmission of the lipid macromolecules was expressed by surface pressure-area measurements per molecule $(\pi-a)$ for aqueous salt subphases. We took advantage of the subphase surface and interface head groups in this experiment to examine interaction at the interface. Monoolein lipid spread from 0.01 M to 0.1 $\mathrm{M}$ across various salt sub-phases and observed their phase transition in molar concentration. $\mathrm{NaCl}, \mathrm{KCl}, \mathrm{KH}_{2} \mathrm{PO}_{4},\left(\mathrm{NH}_{4}\right)_{2} \mathrm{~S}_{2} \mathrm{O}_{8}$, and $\left(\mathrm{NH}_{4}\right)_{2} \mathrm{SO}_{4}$ were the subphase salts used for lipid 
interactions. Extension of $\pi$-a isotherms mean a molecular area was used to find the compressibility parameters for the miscibility of lipid-salt interaction at the interface.

\section{Material and Methods}

\subsection{Materials}

Monoolein lipid purchased from the TCI Chemicals Pvt. Ltd., Chennai, India. The molecular weight of monoolein lipid with an empirical formula of $\mathrm{C}_{21} \mathrm{H}_{40} \mathrm{O}_{4}$ was $356.55 \mathrm{~g} / \mathrm{mol}$ in molecular weight, and the boiling point was $238-240{ }^{\circ} \mathrm{C}$ with a density of $0.94 \mathrm{~g} \mathrm{~cm}^{-3}$ density[27]. Hexane and chloroform were used as spreading spectroscopic grade lipid solvent, purchased from Finar Chemicals Ltd., Ahmedabad, India. Acetone and ethanol were purchased from Rankem Laboratory Reagent, Gurugram, India. The numerous manufacturers of laboratory reagents purchased $\mathrm{NaCl}, \mathrm{KCl}, \mathrm{KH}_{2} \mathrm{PO}_{4},\left(\mathrm{NH}_{4}\right)_{2} \mathrm{~S}_{2} \mathrm{O}_{8}$, and $\left(\mathrm{NH}_{4}\right)_{2} \mathrm{SO}_{4}$. At room temperature $20 \pm 3^{\circ} \mathrm{C}$, the surface tension of the pure aqueous solution was $72.4 \pm 0.3 \mathrm{mN} / \mathrm{m}$. Deionized ultrapure water $\left(18.2 \mathrm{M} \Omega \mathrm{cm}^{-1}, 0.22 \mu \mathrm{m}\right.$ filtration, Millipore, ELIX, Bangalore, India) was used for all experiments. Within three weeks of sample preparation, all isotherms were measured to minimize the effects of solvent evaporation and lipid oxidation.

\subsection{Methods}

\subsubsection{Surface pressure measurements}

The surface pressure measurement was carried out on a fully automatically customized Langmuir Blodgett trough (APEX Instruments Co. Pvt. Ltd., India). The total trough area is 26 $\mathrm{cm} \times 15 \mathrm{~cm}$. The surface pressure v/s area per molecule isotherms was obtained for different subphases by a Wilhelmy plate pressure sensor [5,29]. Teflon trough was thoroughly cleaned with acetone twice before each experimental run, followed by rinsing with Milli-Q water. The purity of the subphase has been verified by ensuring no increase in the surface pressure during barrier compression until the solution is distributed. The subphase in the trough was either Milli-Q water or dissolved salt solutions of $\mathrm{NaCl}, \mathrm{KCl}, \mathrm{KH}_{2} \mathrm{PO}_{4},\left(\mathrm{NH}_{4}\right)_{2} \mathrm{~S}_{2} \mathrm{O}_{8}$, and $\left(\mathrm{NH}_{4}\right)_{2} \mathrm{SO}_{4}$. Each experiment was performed at room temperature $\left(20 \pm 1{ }^{\circ} \mathrm{C}\right)$ in the winter season of the Indian country. The effective surface area of the trough was controlled from both sides by moving a pair of Derlin barriers. An illustrative schematic of the Langmuir trough has been shown in Figure 2. Monoolein lipid was used for spreading on the interface and given enough 
time to evaporate volatile organic solvent for the optimal level of adsorption of macromolecules on the subphase.

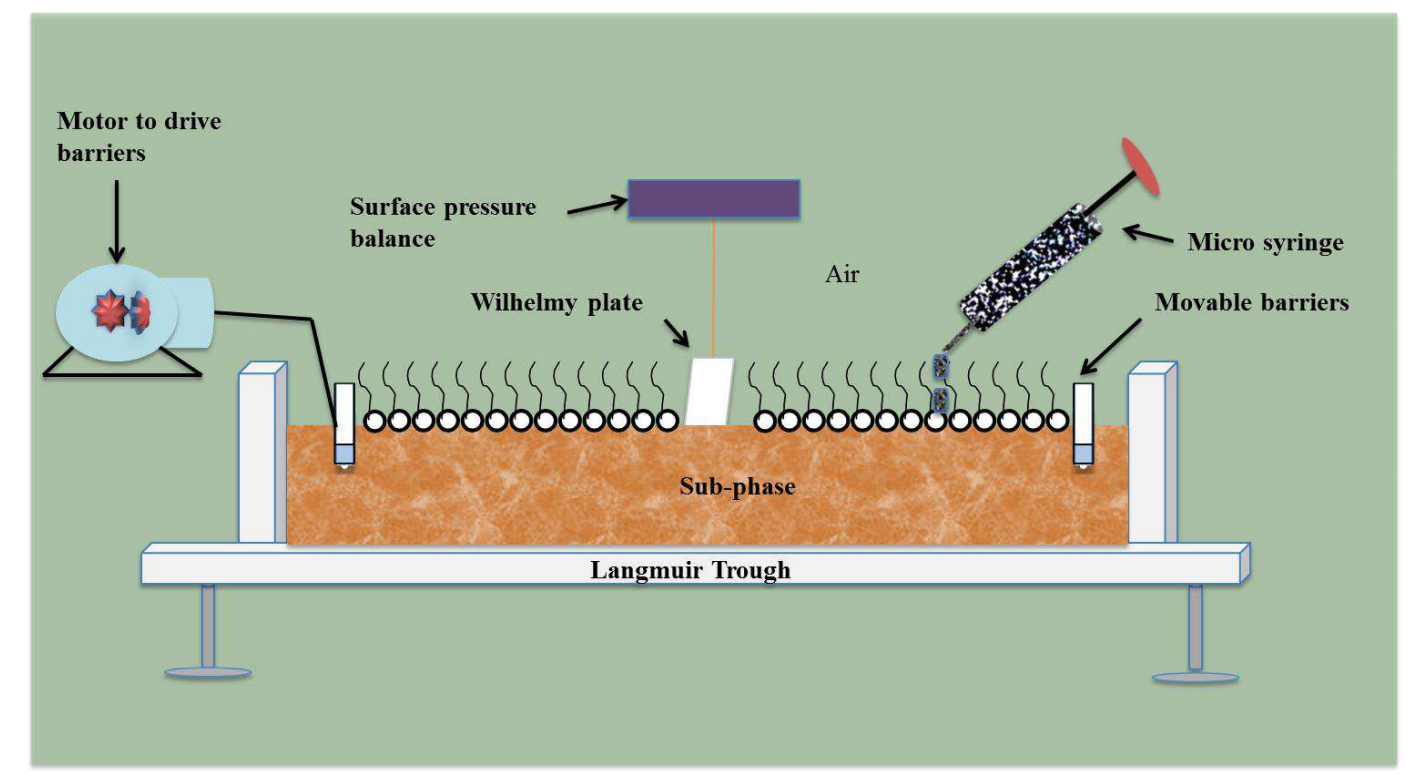

Figure 2. Schematic of the Langmuir monolayer trough diagram with movable barriers

\subsection{Molecular stability of monolayer phases}

Langmuir monolayer approach analysis to investigate the surface properties of lipid macromolecules was conducted at the air-water interface. Langmuir monolayer formation involves the molecule spreading across the surface of the subphase using a volatile organic solvent. As the organic solvent evaporates within 30 min waiting time, molecules at the interfaces achieve a system equilibrium state. The stock solution was prepared by 10 percent chloroform:hexane (v/v) and macromolecules spreading carried using the traditional Trurnit method [30,31]. A gas-tight Hamilton micro-syringe was used to carry out the spreading of molecules. During preliminary trials, the required optimal spreading volumes and spreading concentrations were standardized. The two barriers, which were moving symmetrically, were operated at $1 \mathrm{~mm} / \mathrm{min}$. A standard monolayer experiment is appropriate to spread a few micrograms of spreading solution compounds on a subphase. The resulting compact monolayer film is stable for an extended period if the surface pressure is not too high.

The monolayer will provide the information on two-dimensional surface behaviours and immediate access to the disorder to an organized, ordered layer of confirmations of physical state transmission under compression isotherm. The phase transition sequence, under compression, follows the order of a gas-like, liquid-like, and solid-like monolayer formation at 
the air-water interface, in phase from right to left, respectively. In addition, during the Langmuir isotherm process, the compact macromolecule ensures the hydrophobic-head and hydrophilic-tails of present molecules. Performing the same tests confirmed repeatability and reproducibility. Macromolecules need to be an experiment in the form of a single thin layer for the interaction with external media shown in the schematic diagram below Figure 3 .

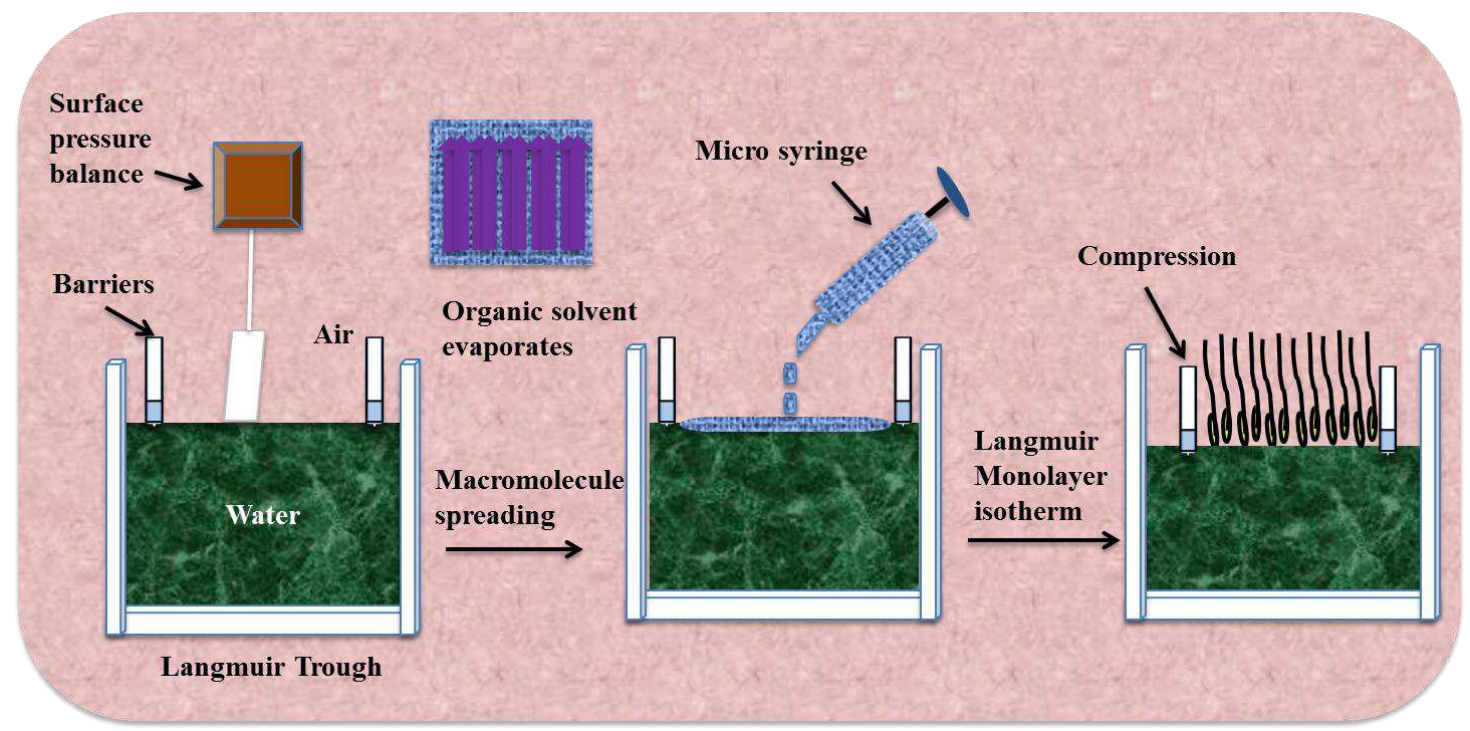

Figure 3. The schematic diagram for monolayer formation at the air-liquid interface

The spreading will continue until the surface pressure has elevated to an equilibrium value until the colliding surface pressure. The colliding surface pressure is defined as when the formed Langmuir monolayer breaks and indicates the sudden shift in surface pressure value towards the reduction. The spreading pressure of the equilibrium is defined as that produced spontaneously when the solid bulk is put in contact with a surface of the water. Again, the vapor pressure of a bulk solid will disburse compression. In the presence of its vapor, an equilibrium vapor pressure occurs for the solid. If this vapor pressure reaches, as the vapor is over-saturated, a deposit on the solid surface will occur. In specific experiments, however, relatively stable surface pressure can be calculated up to higher values than the spreading pressure of the equilibrium [32].

\section{Results and Discussion}

In this contribution, the surface properties of monoolein lipid macromolecules, the single molecules Langmuir monolayer, were developed using the Wilhelmy plate pressure sensor on the air-water interface for the interaction of different salt subphases. The Langmuir monolayer confirms different transition phase positioning, such as gas-like, liquid-like, and solid-like 
behavior on compression isotherm. It is recognized by Langmuir monolayer that the force, which causes the spreading of lipid on the surface of water subphase, was due to the attraction or repulsion of interacting lipid molecules and also surface activity of monoolein lipid. Using the equilibrium surface pressure compression isotherm results, the compressibility calculation was carried out with the mean molecular area of the isotherm. Therefore, an active group of lipid molecules has a marked affinity for spreading the uniform single layer molecule on the subphase.

\subsection{Effect of subphase on monoolein lipid Langmuir monolayer}

Compression isotherm [surface pressure $(\pi)$ - area per molecule (a)] ( $\pi$-a) study allows us to determine the surface pressure increase as a function of decreasing surface area with compression, illustrating the monolayer packing at the interface shown below Figure 4(a). The pure monoolein lipid isotherm was observed to undergo a sharp transition at a molecular area of $\sim 0.88-0.17 \mathrm{~nm}^{2}$, consistent with previous studies [1,27,33]. Due to the compactness of the molecules on barrier compression and also the phenomenon of liftoff surface pressure observed for monoolein lipid monolayer has been shown in Figure 4(a). On the experimental side, the values of salt-free monoolein experimental studies for the area per lipid for monoolein monolayers were recorded for various temperature ranges [33-35]. To validate, our observed value for the area per lipid is therefore in good agreement with the available experimental data at room temperature [34].

In the gaseous phase, lipid molecules' initial compression implies that molecules don't interact with each other. The lipid molecules come closer at further compression, and an increase in surface pressure was observed in Figure 4(a). The monolayer is still in the gaseous phase at this point, thus assuming there was no interaction. This surface pressure increases until an inflection is denoted as the liquid expanded lipid monolayer phase. After more compression with the use of the interfaces, attached barriers move progressively to a liquid condensed phase and then to a solid phase in which the third phase of transition is observed. It means, because of the presence of hydrogen bonds, the liquid condensation phase induces the van der Walls interaction, and the intermolecular interaction phase has been established. The maximum surface pressure inflection has been observed for the pure water subphase at $45.5 \mathrm{mN} / \mathrm{m}$ for the monoolein lipid monolayer shown in Figure 4(a). Following the determination of maximum surface pressure, further compression of lipid monolayer breaks horizontally in the isotherm, implying the collapse of monolayer surface pressure. 
Using different salt subphases in monolayer formation observed the effect of interaction on monoolein lipids at the air-water interface and to identify the effect of lipid monolayer on mono valiant and divalent changes in the distribution of solvent at interfaces. In addition, lipids are weakly interacting with monovalent salts and have a strong interaction with divalent salts [36]. Effects from observations shown in Figure 4(b-f), for the compression isotherm, was the lipid macromolecule spreading on various ionic salt subphase compression isotherm at the airwater interface. It forms a thin layer on the interface after lipid spread due to the inbuilt hydrophobicity nature, making them stay on interfaces. The compression isotherm occurs from the initial liftoff of molecules starting from $0.88 \mathrm{~nm}^{2}$ shown in Figure 4 for different condensed individual salt subphases inflection. In addition, compression of two-dimensional homogenous lipid molecules on the interface changes the disorder of the gas-like phase to liquid expansion arrangements such as physical state since this transition does not occur at constant surface pressure. For all the salt subphases, the compression isotherms liquid transition surface pressure is observed at $5-15 \mathrm{mN} / \mathrm{m}$. Finally, to increase the salt subphase concentration from 0.01 to $0.1 \mathrm{M}$, the collapse phase/solid transition surface pressure for monoolein lipids was measured at approximately 35 to $40 \mathrm{mN} / \mathrm{m}$. It determined the maximum surface pressure by which the highest number of molecules is adsorbed on the surface.

In the monolayer, the interactions between components can be investigated through the excess area from the miscibility between components in the subphase. These results suggest that higher surface pressures are dominated by salt solution. We represented in Figure 4(a) hypothetical $\pi$-a isotherms for compression monolayer calculated for lipid molecules on pure water subphase is present at the air-water interface. Likewise, other salt's compression isotherm plots were shown in Figure 4(b-f). Similarly, they were observed for change of physical state on compression with simultaneous moving barriers. The observed physical state was a gaslike, liquid-like, and solid-like monolayer on the surface pressure value of $5 \mathrm{mN} / \mathrm{m}, 30 \mathrm{mN} / \mathrm{m}$, and $35 \mathrm{mN} / \mathrm{m}$ on the air-water interfaces, respectively. In addition, the area per molecule of 0.9 to $0.7 \mathrm{~nm}^{2}$ for gas-like, 0.7 to $0.4 \mathrm{~nm}^{2}$ for liquid-like, and 0.4 to $0.17 \mathrm{~nm}^{2}$ for solid-like monolayer were measured at interfaces with the various salt subphases for monoolein Langmuir monolayer formation. On the surface as well as on the interface, the main parameters affecting the analysis were distinguished: $\mathrm{pH}$ scale, ionic strength, charge density, concentration, surface activity, structure, miscibility, superficial viscosity, film formation 
kinetics at fluid interfaces, etc., and biopolymer penetration on the insoluble subphase interfaces [37].

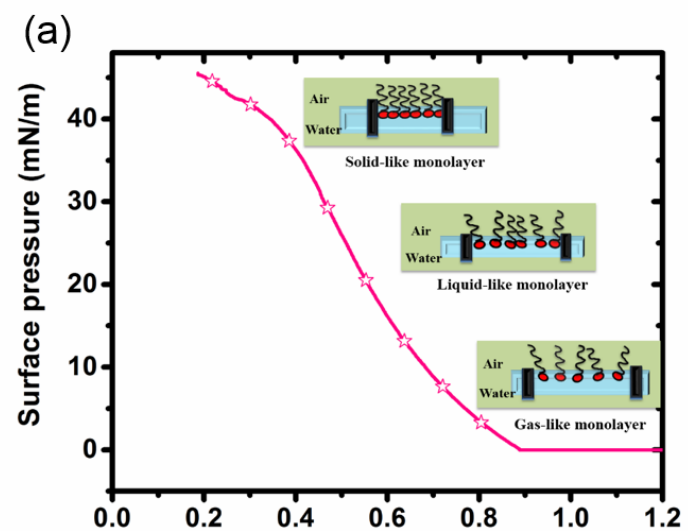

(b)

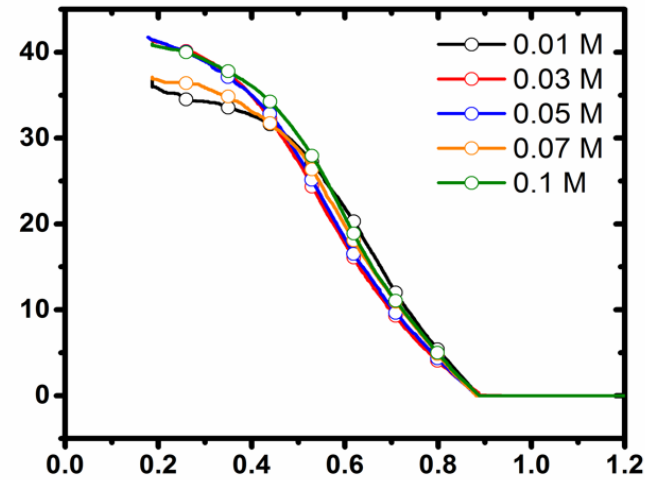

(c)

(d)
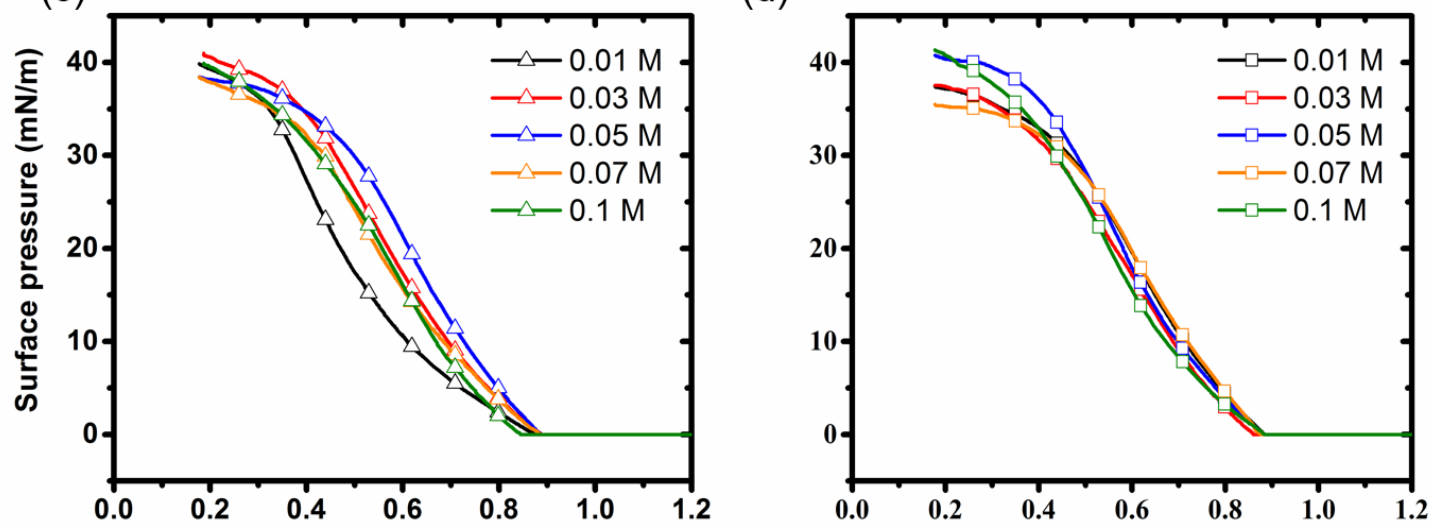

(e)

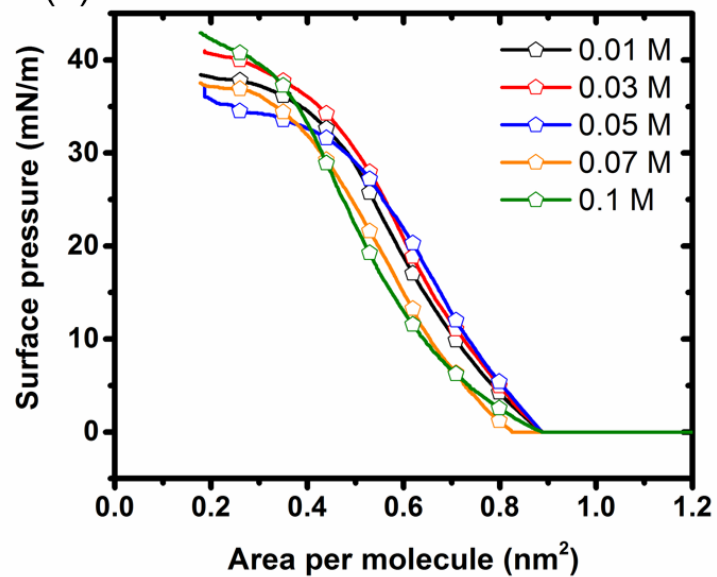

(f)

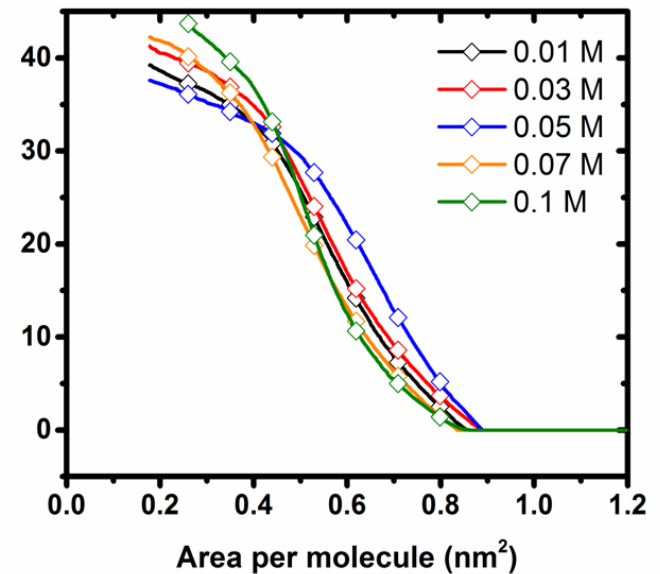

Figure 4. Effect of compression isotherm of the monoolein lipid on different salt subphases at the airwater interface, (a) water subphase, (b) $\mathrm{NaCl}$ salt solution subphase, (c) $\mathrm{KCl}$ salt solution subphase, (d)

$\mathrm{KH}_{2} \mathrm{PO}_{4}$ salt solution subphase, (e) Ammonium sulphate salt solution subphase, (f) Ammonium persulphate salt solution subphase.

\subsection{Determination of surface compressibility modulus}

Figure 5(a-e) demonstrates the measured compressibility and elastic module for the previously obtained Langmuir isotherm data in various experiments under the change in salt 
concentrations for the formation of lipid Langmuir monolayer. The compressibility and elastic modulus were computed using the following eq. 1 . The mean molecular area obtained by means of compression isotherm is respectively. Many researchers conduct the compressibility modulus recognition based on eq. 1 to select parameters over the lipid monolayer's physical state, in their extension analysis towards the Langmuir isotherm [38,39]. The information provided by the $\pi$-A isotherm, the compressibility $C_{s}$, was determined to be evaluated in more detail.

$$
C_{S}^{-1}=-\frac{1}{A}\left(\frac{\partial \pi}{\partial A}\right)_{T}
$$

Whereas, A denotes the mean molecular area, $\pi$ - surface pressure, is at room temperature $\mathrm{T}$ (approx $20{ }^{\circ} \mathrm{C}$ ). From the experimental observation, shown the compressibility modulus is $C_{S}$ $<10 \mathrm{mN} / \mathrm{m}$ for liquid expanded (LE) state, $10-25 \mathrm{mN} / \mathrm{m}$ for liquid condensed (LC) state, $>25$ $\mathrm{mN} / \mathrm{m}$ for solid (S) state. These experimental findings for the compressibility modulus are consistent with the results previously published [22]. Also, their LE state did not start from the zero surface pressure, and then it decreased, as we observed in Figure 5. With the shift in slope values, the physical state transitions behaviour can be discovered from the $C_{s}-\pi$ curve study. In addition, $C_{S}$, according to the Davies and Rideal description, offers information on the monolayer phases and phase transitions physical state [40].

However, lipid surfactants have attracted a lot of attention at the air-water interface to create a Langmuir monolayer to monitor the molecular structure interface. In addition, combinations of various subphase solutions are employed to understand both the fundamental knowledge of lipid interaction and the monolayer formation influence, as well as the role of net loading, etc. [39,40]. A derived compressibility equation of the modulus is defined using the average mean molecular area of the monolayer at the air-water interface to deduce the physical state and orientation of the formed monolayer Langmuir. For monooline lipid, the compression of the lipid spreading isotherm $(\pi-\mathrm{A})$, which occurs at the lowest level of the $C_{s}^{-1}$ surface pressure isotherms, which occurs at the lowest level of the $C_{s}^{-1}$ surface pressure isotherm, was observed on the plateau of transitional changes in gas-like, fluid-like and solidlike behaors. The obtained $C_{s}^{-1}$ values were below $50 \mathrm{mN} / \mathrm{m}$, indicating a liquid expanded phase, and a change in the phase-state of the lipid on the air-water interface, was not due to any change of the $\pi$-A isotherms [41]. Even at low surface pressures, the compressibility module is not directly linked with its true rheological and shear properties [42]. 

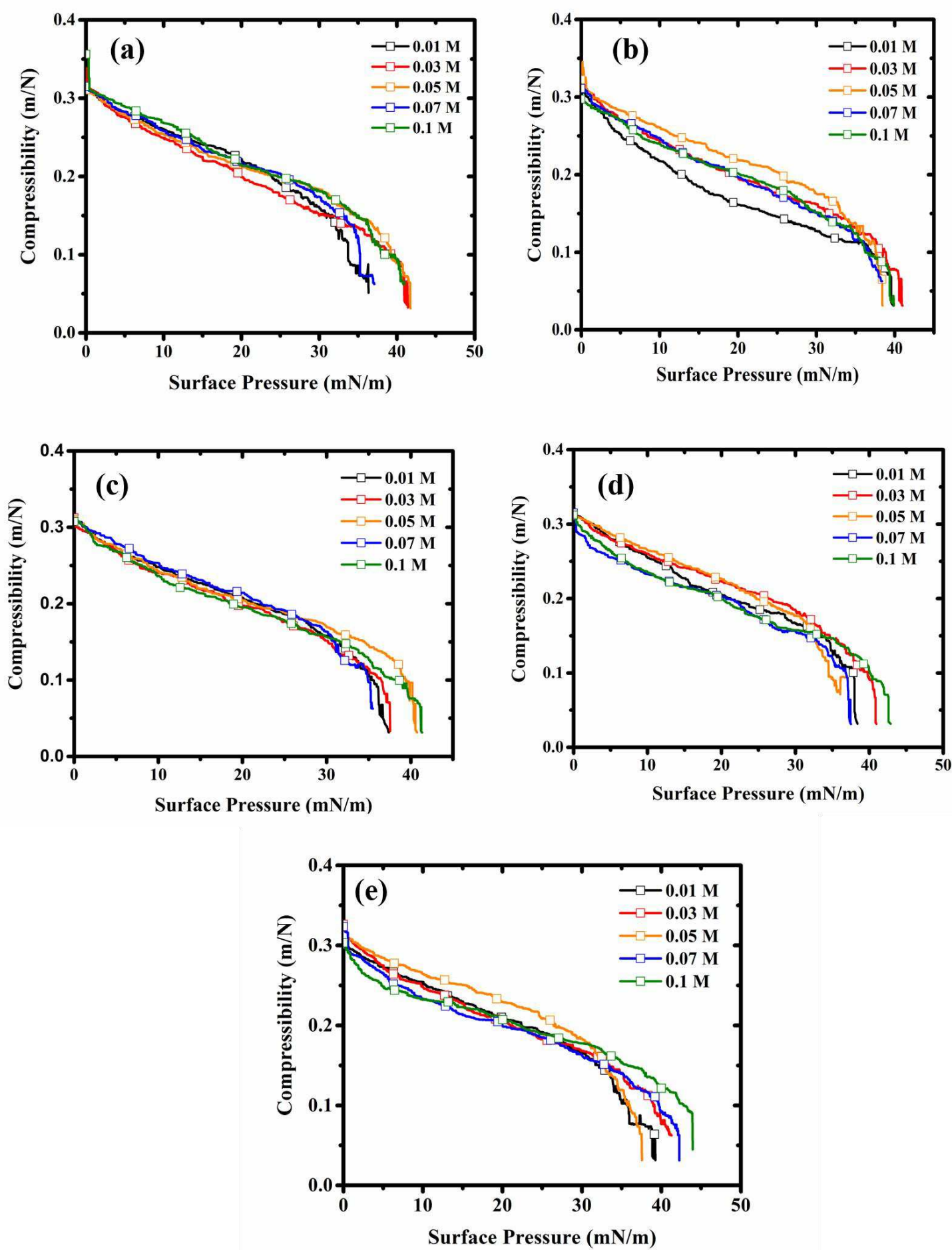

Figure 5. Effect of the compressibility of monoolein lipid monolayers on a surface with the change in different salt subphases at the air-water interface, (a) NaCl salt subphase, (b) KCl salt subphase, (c) $\mathrm{KH}_{2} \mathrm{PO}_{4}$ subphase, (d) ammonium sulphate salt subphase (e) ammonium persulphate salt subphase.

The range of the value of $C_{s}^{-1}$ depends on the specific system being characterized. The compression isotherm $(\pi-\mathrm{A})$ from a lipid monolayer describe various salt subphases in a liquid condensed state $(\pi>25 \mathrm{mN} / \mathrm{m})$, a drop-in $C_{s}^{-1}$ values in $\mathrm{NaCl}$ and $\mathrm{KCl}$, but the case of $\mathrm{KH}_{2} \mathrm{PO}_{4}$, 
$\left(\mathrm{NH}_{4}\right)_{2} \mathrm{~S}_{2} \mathrm{O}_{8}$, and $\left(\mathrm{NH}_{4}\right)_{2} \mathrm{SO}_{4}$, no substantial change in $C_{s}^{-1}$ values was observed. The introduction of $\mathrm{NaCl}$ or $\mathrm{KCl}$ into the monolayer phospholipids in the liquid-condensed state indicates that a more liquid extended surface layer is developed [43]. The Golden rule says that the less the compression module value is, the more the monolayer is compressible [44]. Furthermore, the overall compressibility values can help to improve lipid monolayer stability. When adding salt to the subphase of lipid monolayers causes a reduction in the values of $C_{s}^{-1}$, it means that the salt and the monolayer components may be partly miscible.

The well-formed physical state displays the order of the gas, liquid, liquid-expanded to liquid-condensed, and in the end solid phase transition state in sequential order. But sometimes, the distribution of homogeneous layer, penetration time, and size of the molecule is deformed so that they are no longer in a circular shape [45]. These superficial structural characteristics might cause our monoolein lipid, which shows only one transition phase, the acceptable version of a phase transition asymmetric peak consisting of one single step at least, as shown in Figure 5. However, the effect is different for subphase conditions used on interfacial interactions of a monoolein lipid monolayer. More data on the dynamic and elastic properties and the application of $C_{s}^{-1}$ study on membrane lipids and their mixtures can be found in several studies [4,46-48]. The presence of interacting forces is almost the same in the compression equation as surface pressure $\alpha$-a isotherms measurements. The slit complement to the interactions between van der Waals and the hydrocarbon chain of the lipid and hydrogen bond links at the interface form the network with the lipid polar head group. This enhances the attraction or repulsion of the uniform distributive single-molecule lipid layer, particularly the affinity to change the subphase of salt in the bulk aqueous process [35,49]. It may also be associated in the bulk aqueous phase with monolayer molecular loosening by collapse and dissolution, as seen for the same lipid propagation to the aqueous solution [50].

With salt surface groups and lipid heads being loaded, electrostatic interactions seem highly likely to be the driving force, but interactions with the lipid tail might justify all this. This could allow the salt to penetrate the lipid layer deeper and, therefore, significantly higher surface pressure changes than other molar concentrated subphases in $0.1 \mathrm{M}$ salt forms. Figure $5(\mathrm{a}-\mathrm{f})$ shows that higher concentrations of $0.1 \mathrm{M}$ salt have resulted in monoolein interaction, while monoolein monolayer binding did not affect this. Interestingly, the anionic salt subphase demonstrated substantially greater interaction with both monolayers at high concentrations 
than the cationic salt subphase. Since Na ions are almost not bound, the high concentration of sodium does not directly affect the density of the surface. Still, a high level of $\mathrm{Na}^{+}$ion will indirectly affect the density of surface charges. Thus, their impact on the surface concentration of lipids, in particular by an increase in $\mathrm{Na}^{+}$ion concentration [14]. However, this study can help develop finished products and determine the physicochemical properties of a stable system. The main goal is to connect different possible subphase interaction behaviours to understand the fundamental research behind the interactive lipid interface affected by other molecules at the air-water interface. 


\section{Conclusion}

Using the Langmuir monolayer technique on air-water interfaces, we observed the surface properties of various salt subphases and their change in concentration for the spreading of monoolein lipid macromolecules at interfaces. Characterizes the effect of sub-phase ions on the monoolein lipid monolayer 's phase behaviour, conformation, and head group structure. The inflection observed for monoolein lipid surface pressures was $45.5 \mathrm{mN} / \mathrm{m}$ for pure water subphase at the air-water interface. Collapse pressure for lipid is about $40 \mathrm{mN} / \mathrm{m}, 39 \mathrm{mN} / \mathrm{m}$, $40 \mathrm{mN} / \mathrm{m}, 37 \mathrm{mN} / \mathrm{m}$, and $42 \mathrm{mN} / \mathrm{m}$ for the change in salt subphase types. The concentrated salt subphases of $\mathrm{NaCl}, \mathrm{KCl}, \mathrm{KH}_{2} \mathrm{PO}_{4},\left(\mathrm{NH}_{4}\right)_{2} \mathrm{~S}_{2} \mathrm{O}_{8}$, and $\left(\mathrm{NH}_{4}\right)_{2} \mathrm{SO}_{8}$ have shown feasible data analysis at the air-water interface. During compression of lipid monolayer, the $\pi$-a isotherms reveal high accuracy on the change in transition phases and disorder the lateral molecule packing disordered molecules to ordered molecules at the interfaces for all the solutions. The modulus of compression is used to gain knowledge of the miscibility of lipid-salt molecules. $C_{s}^{-1}$ offers insights on the monolayer system and responds better during lateral interactions to slight changes in the monolayer structure. Preliminary studies of lipid monolayer interactions are essential for understanding the organized relationship of function to various areas, such as ion transportation, photosynthesis, cell recognition, and numerous disease conditions. The Langmuir lipid monolayer must be established and monitored in multiple food systems to ensure improved structural stability, its physicochemical properties, macrostructure, and the resulting rheological surface characteristics.

\section{Acknowledgments}

The authors thank the Ministry of Human Resource Development (MHRD), Government of India, for providing fellowship to BD, and SVNIT-Surat for kind support for providing the facilities of the research work.

\section{Conflict of interest}

The authors declare that there is no conflict of interest.

Ethical approval This article does not contain any studies with human participants or animals performed by any authors.

\section{References}


[1] C. Carrera Sánchez, M. Cejudo Fernández, M. Rosario Rodríguez Niño, J. M. Rodríguez Patino, Thermodynamic and Dynamic Characteristics of Monoglyceride Monolayers Penetrated by $\beta$-Casein, Langmuir. 22 (2006) 4215-4224. doi:10.1021/la053506+.

[2] S. Gallier, D. Gragson, R. Jiménez-Flores, D.W. Everett, $\beta$-Casein-phospholipid monolayers as model systems to understand lipid-protein interactions in the milk fat globule membrane, Int. Dairy J. 22 (2012) 58-65. doi:https://doi.org/10.1016/j.idairyj.2011.08.007.

[3] S.S. Ali, X. Tang, S. Alavi, J. Faubion, Structure and Physical Properties of Starch/Poly Vinyl Alcohol/Sodium Montmorillonite Nanocomposite Films, J. Agric. Food Chem. 59 (2011) 12384-12395. doi:10.1021/jf201119v.

[4] M. Elderdfi, A.F. Sikorski, Langmuir-monolayer methodologies for characterizing proteinlipid interactions, Chem. Phys. Lipids. 212 (2018) 61-72. doi:10.1016/j.chemphyslip.2018.01.008.

[5] B. Dhopte, V.N. Lad, Alteration of surface pressure of macromolecular monolayer at the airwater interface and electrochemical impedance characteristics, Colloids Surfaces B Biointerfaces. 187 (2020) 110638. doi:10.1016/J.COLSURFB.2019.110638.

[6] J. young Park, R. Advincula, Nanostructuring polymers, colloids, and nanomaterials at the airwater interface through Langmuir and Langmuir-Blodgett techniques, Soft Matter. 7 (2011) 9829-9843. doi:10.1039/C1SM05750B.

[7] Y.D. Livney, Milk proteins as vehicles for bioactives, Curr. Opin. Colloid Interface Sci. 15 (2010) 73-83. doi:10.1016/j.cocis.2009.11.002.

[8] G. Thakur, M. Micic, R.M. Leblanc, Surface chemistry of Alzheimer's disease: a Langmuir monolayer approach., Colloids Surf. B. Biointerfaces. 74 (2009) 436-456. doi:10.1016/j.colsurfb.2009.07.043.

[9] J.M. Rodríguez Patino, M.C. Fernández, Structural and Topographical Characteristics of Adsorbed WPI and Monoglyceride Mixed Monolayers at the Air-Water Interface, Langmuir. 20 (2004) 4515-4522. doi:10.1021/la036190j.

[10] M. Rojewska, M. Skrzypiec, K. Prochaska, Surface properties and morphology of mixed POSS-DPPC monolayers at the air/water interface, Colloids Surf. B. Biointerfaces. 150 (2017) 334-343. doi:10.1016/j.colsurfb.2016.10.047.

[11] A.S. Chumakov, A. V Ermakov, I.A. Gorbachev, E.L. Kossovich, A.A. Kletsov, E.G. Glukhovskoy, The influence of redistribution ions in subphase at the properties Langmuir monolayer: physical and theoretical experiments, in: Proc.SPIE, 2016. https://doi.org/10.1117/12.2228787.

[12] D. Tauraite, V. Razumas, T. Nylander, E. Butkus, Interfacial behavior of ferrocene-and 1, 4naphthoquinone-based compounds, and their mixtures with monoolein at the air/water interface, Zeitschrift Für Naturforsch. B. 63 (2008) 1093-1100.

[13] E. Jabłonowska, E. Nazaruk, D. Matyszewska, C. Speziale, R. Mezzenga, E.M. Landau, R. Bilewicz, Interactions of Lipidic Cubic Phase Nanoparticles with Lipid Membranes, Langmuir. 32 (2016) 9640-9648. doi:10.1021/acs.langmuir.6b01746.

[14] R. Kensbock, H. Ahrens, C.A. Helm, Interactions of Monovalent and Divalent Cations with Cardiolipin Monolayers, Langmuir. 35 (2019) 3624-3633. doi:10.1021/acs.langmuir.8b03637.

[15] J.A. O’Mahony, P.F. Fox, Milk: An Overview, Milk Proteins. (2014) 19-73. doi:10.1016/B978-0-12-405171-3.00002-7.

[16] E. Dickinson, D.J. McClements, Advances in food colloids, Springer Science \& Business Media, 1995.

[17] N.J. Krog, F.V. Sparso, Food emulsifiers and their chemical and physical properties, Marcel Dekker Inc., New York, USA, 2004.

[18] M. Rosario Rodríguez Niño, P. J. Wilde, D. C. Clark, J. M. Rodríguez Patino, Surface Rheological Properties of Monostearin and Monoolein Films Spread on the Air-Aqueous Phase Interface, Ind. \&amp; Eng. Chem. Res. 35 (1996) 4449-4456. doi:10.1021/ie960333y.

[19] J. de la Fuente Feria, J. M. Rodriguez Patino, Destabilization of Monoglyceride Monolayers at the Air-Aqueous Subphase Interface. 1. Kinetics, Langmuir. 10 (2002) 2317-2324. doi:10.1021/la00019a049.

[20] M.J. Hostetler, S.J. Green, J.J. Stokes, R.W. Murray, Monolayers in three dimensions : synthesis and electrochemistry of $\omega$-functionalized alkanethiolate-stabilized gold cluster 
compounds, J. Am. Chem. Soc. 118 (1996) 4212-4213.

[21] D. Lee, O. Redfern, C. Orengo, Predicting protein function from sequence and structure, Mol. Cell Biol. 8 (2007) 995-1005. doi:10.1038/nrm2281.

[22] R.P. Giri, M.K. Mukhopadhyay, M. Mitra, A. Chakrabarti, M.K. Sanyal, S.K. Ghosh, S. Bera, L.B. Lurio, Y. Ma, S.K. Sinha, Differential adsorption of a membrane skeletal protein, spectrin, in phospholipid membranes, EPL (Europhysics Lett. 118 (2017) 58002. doi:10.1209/0295-5075/118/58002.

[23] R. P. Giri, A. Chakrabarti, M. K. Mukhopadhyay, Cholesterol-Induced Structural Changes in Saturated Phospholipid Model Membranes Revealed through X-ray Scattering Technique, J. Phys. Chem. B. 121 (2017) 4081-4090. doi:10.1021/acs.jpcb.6b12587.

[24] T.-B. J, R. R, Effect of lysozyme subphase and insertion on several lipid films, Adv. Mater. Sci. 4 (2019) 1-7. doi:10.15761/AMS.1000149.

[25] L. Zhang, Q. Lu, M. Liu, Fabrication of Chiral Langmuir-Schaefer Films from Achiral TPPS and Amphiphiles through the Adsorption at the Air/Water Interface, J. Phys. Chem. B. 107 (2003) 2565-2569. doi:10.1021/jp026621r.

[26] B. Piknova, V. Schram, S.B. Hall, Pulmonary surfactant: phase behavior and function., Curr. Opin. Struct. Biol. 12 (2002) 487-494. doi:10.1016/s0959-440x(02)00352-4.

[27] C. V Kulkarni, W. Wachter, G. Iglesias-Salto, S. Engelskirchen, S. Ahualli, Monoolein: a magic lipid?, Phys. Chem. Chem. Phys. 13 (2011) 3004-3021. doi:10.1039/C0CP01539C.

[28] L. Ariza-Carmona, M.T. Martín-Romero, J.J. Giner-Casares, M. Pérez-Morales, L. Camacho, Elastic Nanocomposite Structures Formed by Polyacetylene-Hemicyanine Mixed Films at the Air-Water Interface, J. Phys. Chem. C. 117 (2013) 21838-21848. doi:10.1021/jp406397q.

[29] D.M. Taylor, G.F. Bayes, The surface potential of Langmuir monolayers, Mater. Sci. Eng. C. C (1999) 65-71.

[30] H.J. Trurnit, A theory and method for the spreading of protein monolayers, J. Colloid Sci. 15 (1960) 1-13. doi:https://doi.org/10.1016/0095-8522(60)90002-7.

[31] R.S. Phadke, G. Agarwal, Methods of deposition and characterization of thin films of amphiphiles, Mater. Sci. Eng. C. C (1999) 113-118.

[32] C.C. Sánchez, M.C. Fernández, M.R. Rodríguez Niño, J.M. Rodríguez Patino, Thermodynamic and Dynamic Characteristics of Monoglyceride Monolayers Penetrated by $\beta$ Casein, Langmuir. 22 (2006) 4215-4224. doi:10.1021/la053506+.

[33] J.M. Rodriguez Patino, M. Cejudo Fernandez, C. Carrera Sanchez, M.R. Rodriguez Nino, Structural and shear characteristics of adsorbed sodium caseinate and monoglyceride mixed monolayers at the air-water interface., J. Colloid Interface Sci. 313 (2007) 141-151. doi:10.1016/j.jcis.2007.04.025.

[34] A.A. Gurtovenko, I. Vattulainen, Membrane Potential and Electrostatics of Phospholipid Bilayers with Asymmetric Transmembrane Distribution of Anionic Lipids, J. Phys. Chem. B. 112 (2008) 4629-4634. doi:10.1021/jp8001993.

[35] J.M. Rodríguez Patino, M.R. Rodríguez Niño, C. Carrera Sánchez, Structure, Miscibility, and Rheological Characteristics of $\beta$-Casein-Monoglyceride Mixed Films at the Air-Water Interface, J. Agric. Food Chem. 51 (2003) 112-119. doi:10.1021/jf020197+.

[36] A. Honigmann, G. van den Bogaart, E. Iraheta, H.J. Risselada, D. Milovanovic, V. Mueller, S. Müllar, U. Diederichsen, D. Fasshauer, H. Grubmüller, S.W. Hell, C. Eggeling, K. Kühnel, R. Jahn, Phosphatidylinositol 4,5-bisphosphate clusters act as molecular beacons for vesicle recruitment, Nat. Struct. \&Amp; Mol. Biol. 20 (2013) 679. https://doi.org/10.1038/nsmb.2570.

[37] R. Ettelaie, A. Akinshina, Colloidal interactions induced by overlap of mixed protein $p$ polysaccharide interfacial layers, Food Hydrocoll. 42 (2014) 106-117. doi:10.1016/j.foodhyd.2014.01.020.

[38] S. Chitra, P. Bargavi, M. Balasubramaniam, R.R. Chandran, S. Balakumar, Impact of copper on in-vitro biomineralization, drug release efficacy and antimicrobial properties of bioactive glasses, Mater. Sci. Eng. C. 109 (2020) 110598. doi:https://doi.org/10.1016/j.msec.2019.110598.

[39] J. Torrent-Burgués, P. Cea, I. Giner, E. Guaus, Characterization of Langmuir and LangmuirBlodgett films of an octasubstituted zinc phthalocyanine, Thin Solid Films. 556 (2014) 485494. doi:10.1016/J.TSF.2014.01.045. 
[40] J.T. Davies, Interfacial phenomena, Elsevier, 2012.

[41] R. Maget-Dana, The monolayer technique: a potent tool for studying the interfacial properties of antimicrobial and membrane-lytic peptides and their interactions with lipid membranes., Biochim. Biophys. Acta. 1462 (1999) 109-140. doi:10.1016/s0005-2736(99)00203-5.

[42] B. Caruso, E.E. Ambroggio, N. Wilke, G.D. Fidelio, The rheological properties of beta amyloid Langmuir monolayers: Comparative studies with melittin peptide, Colloids Surfaces B Biointerfaces. 146 (2016) 180-187. doi:10.1016/J.COLSURFB.2016.06.003.

[43] M.J. Sánchez-Martín, I. Haro, M.A. Alsina, M.A. Busquets, M. Pujol, A Langmuir Monolayer Study of the Interaction of E1(145-162) Hepatitis G Virus Peptide with Phospholipid Membranes, J. Phys. Chem. B. 114 (2010) 448-456. doi:10.1021/jp906900k.

[44] E. Lopez-Rodriguez, J. Pérez-Gil, Structure-function relationships in pulmonary surfactant membranes: From biophysics to therapy, Biochim. Biophys. Acta - Biomembr. 1838 (2014) 1568-1585. doi:https://doi.org/10.1016/j.bbamem.2014.01.028.

[45] J.M. Rodriguez Patino, M.R. Rodriguez Nino, C. Carrera Sanchez, M. Cejudo Fernandez, The Effect of $\mathrm{pH}$ on Monoglyceride-Caseinate Mixed Monolayers at the Air-Water Interface., J. Colloid Interface Sci. 240 (2001) 113-126. doi:10.1006/jcis.2001.7567.

[46] R.E. Brown, H.L. Brockman, Using monomolecular films to characterize lipid lateral interactions, Methods Mol. Biol. 398 (2007) 41-58. doi:10.1007/978-1-59745-513-8_5.

[47] M. Jurak, J. Miñones, Interactions of lauryl gallate with phospholipid components of biological membranes, Biochim. Biophys. Acta - Biomembr. 1858 (2016) 1821-1832. doi:10.1016/J.BBAMEM.2016.04.012.

[48] X. Zhai, X.-M. Li, M.M. Momsen, H.L. Brockman, R.E. Brown, Lactosylceramide: Lateral Interactions with Cholesterol, Biophys. J. 91 (2006) 2490-2500. doi:10.1529/BIOPHYSJ.106.084921.

[49] J. de la Feria, J. M. Rodriguez Patino, Destabilization of Monoglyceride Monolayers at the Air-Aqueous Subphase Interface. 2. The Role of Film Elasticity, Langmuir. 11 (2002) 20902097. doi:10.1021/la00006a041.

[50] C. Carrera Sánchez, J. de la Fuente Feria, J.M. Rodríguez Patino, The importance of the monolayer structure in bidimensional miscibility in mixed films. 1. Kinetics, Colloids Surfaces A Physicochem. Eng. Asp. 143 (1998) 477-490. doi:https://doi.org/10.1016/S09277757(98)00378-1. 


\section{Supplementary Files}

This is a list of supplementary files associated with this preprint. Click to download.

- GF.tif 